\title{
Inter-Laboratory Analysis of Steviol Glycosides by an External Standard Method
}

\author{
Joannes Martinus Cornelius Geuns \\ Laboratory of Functional Biology, KU Leuven, Leuven, Belgium
}

Email address:

Jan.Geuns@bio.kuleuven.be

To cite this article:

Joannes Martinus Cornelius Geuns. Inter-Laboratory Analysis of Steviol Glycosides by an External Standard Method. International Journal of Nutrition and Food Sciences. Vol. 7, No. 5, 2018, pp. 173-179. doi: 10.11648/j.ijnfs.20180705.13

Received: August 29, 2018; Accepted: September 10, 2018; Published: October 11, 2018

\begin{abstract}
To optimise steviol glycoside analysis, several round-robin tests were organised by the European Stevia Association (EUSTAS). Seventeen laboratories participated in the testing. Only 8 laboratories have sent their results. In the first round-robin testing, 2 samples were analysed. The first sample had a purity of $96.2 \%$. The second sample was a $4 / 5$ dilution with $\mathrm{NaHCO}_{3}$ of sample 1. This way, the drying process itself could be checked. The purity of sample 2 was $82.35 \%$. The reported purities of sample 1 varied between 79.8 and $96.2 \%$, those of sample 2 varied between 58.1 and $81.8 \%$. The drying of sample 2 showed that weight loss was between 4.9 and $12.7 \%$, demonstrating that not all laboratories dried the sample to a constant weight. In a second round-robin testing, the purity of the sample was $91.1 \%$. The reported purities of the sample varied between 82.74 and $95.86 \%$. About $3 \%$ purified RebB was added to the sample to check the quality of the analysis of this compound possessing a carboxylic group. The samples contained the following steviol glycosides: Reb D, Reb E, Reb A, ST, Reb F, Reb C, Dul A, Reb G, Rub, Reb B, SB and SM (1 lab). No SV was detected. The number of SVgly analysed in the different laboratories varied between 4 and 11. One lab only analysed ST and Reb A and gave a percentage composition of these compounds. To improve the accuracy of analysis, different suggestions are made, such as controlling the drying process of samples and standards, purity of standards, injection of sufficient material and use of solvent gradients to shorten the run time and to reduce integration errors. The results of this second round-robin tests are better than those of the first one.
\end{abstract}

Keywords: Steviol Glycosides, Round-robin Testing, Validation of Analytical Methods, HPLC

\section{Introduction}

A good validated method of analysis of steviol glycosides is of utmost importance for food inspectors as well as for the Stevia industry. The minimum purity requirement of $95 \%$ by JECFA and EFSA makes the analysis of steviol glycosides a very difficult task, as possible errors should be eliminated or at least be minimized. The result of a sample analysis should always be the same and totally independent of the laboratory that performed the analysis. As shown earlier, the proposed JECFA [3] method is not reliable and many analysts do not use it strictly as described in the protocol $[1,2]$. The analysis of steviol glycosides by HPLC is essentially based on 2 different column types: adsorption (propylamine $\left(-\mathrm{NH}_{2}\right)$; HILIC)) or reversed phases (well selected phases of Octadecyl silica gel $\left(\mathrm{C}_{18}\right)$ ). The column dimensions are usually $250 \times 4.6 \mathrm{~mm}$ and particle size, $5 \mu \mathrm{m}$. Some people use HILIC columns $[1,4,5]$. To obtain a baseline separation, required for perfect peak integration, it might be necessary to use $2 \mathrm{C}_{18}$ columns in series or 1 ultra-high performance liquid chromatography column [6]. More literature on HPLC of steviol glycosides was cited in this last paper, but no baseline separation was obtained or not all the steviol glycosides were included in the analysis [7-10]. Usually, UVdetection at $210 \mathrm{~nm}$ is used, although some laboratories confirm the identity of the different compounds by LC-MS. To increase sensitivity and if the equipment is suited, measurements can be done at a lower wavelength (e.g. 190 $\mathrm{nm})$. Solvents used normally are mixtures of acetonitrile: water $\left(\mathrm{AcCN}: \mathrm{H}_{2} \mathrm{O}\right)$ or $\mathrm{AcCN}$ in combination with diluted NH4OAc or phosphoric acid. With $\mathrm{NH}_{2}$-columns, an isocratic solvent $\mathrm{AcCN}$ : water phase (between 87:13 and $80: 20$ ) is often used. Under these conditions, the run time 
may be as long as $65-75 \mathrm{~min}$.

On $\mathrm{C}_{18}$-type columns, a baseline separation can be obtained by use of 2 Grace Alltima $\mathrm{C}_{18}$ columns in series and a solvent gradient of $\mathrm{AcCN}$ : $0.1 \mathrm{mM}$ phosphoric acid (see below) In theory, after a proper calibration, both types of steviol glycoside analyses should give similar results. The steviol glycoside analysis has previously been validated [11] Recently, a review on analytical methods was published, but no detailed discussion was given on the accuracy of the different methods nor on the use of a method in different laboratories [12]. However, inter-laboratory accuracy should also be tested and therefore round-robin tests were organised.

In our round-robin testings, we advised the use of $\mathrm{C}_{18}$ columns as these can easily be rinsed with different solvents without damaging the columns.
As most of the laboratories do not have all the ultra-pure standards available for calibration, it is usually done only with stevioside and/or rebaudioside A. Previous work has shown that this is satisfactory as most of the steviol glycosides have a similar absorption coefficient [2, 11]. Therefore, the slopes of the calibration curves of all steviol glycosides are very similar as the calibration curves were made with $\mathrm{mM}$ concentrations $[1,2]$. To express the results as weight by weight, (e.g. mg component/100 mg mixture) a correction has to be made for the different molecular masses [1]. Table 1 gives the slopes, molecular masses and conversion factors to obtain concentrations in $\mathrm{mg} / \mathrm{mL}$ if calibration curves were made in $\mathrm{mM}$ concentrations (from [11]).

Table 1. Molecular weights and conversion factors are given to calculate concentrations in $\mathrm{mg} / \mathrm{mL}$ of different steviol glycosides in a mixture after calibration of the HPLC with stevioside $(5 \mathrm{mg} / 5 \mathrm{~mL}=1.243 \mathrm{mM})$, or $\mathrm{RA}$ respectively $(5 \mathrm{mg} / 5 \mathrm{~mL}=1.035 \mathrm{mM})$.

\begin{tabular}{llll}
\hline Compound & Slopes $\mathbf{m} \pm \mathbf{s e}$ & Molecular weights & Conversion Factors $(\mathbf{C F})$ to obtain concentration in mg/mL \\
\hline Stevioside & $4.52 \pm 0.11$ & 804.38 & 0.80438 \\
Rebaudioside A & $4.18 \pm 0.04$ & 966.43 & 0.96643 \\
Rebaudioside C & $4.32 \pm 0.02$ & 950.44 & 0.95044 \\
Dulcoside A & $4.12 \pm 0.04$ & 788.38 & 0.78838 \\
Rubusoside & $4.43 \pm 0.13$ & 642.33 & 0.64233 \\
Steviolbioside & $2.30 \pm 0.05$ & 642.33 & 0.64233 \\
Rebaudioside B & $3.10 \pm 0.11$ & 804.38 & 0.80438 \\
Rebaudioside D & nd & 1128.48 & 1.12848 \\
Rebaudioside E & nd & 966.43 & 0.96643 \\
Rebaudioside F & nd & 936.42 & 0.93642 \\
\hline
\end{tabular}

\section{Method}

EUSTAS Round-Robin testing

Seventeen laboratories agreed to participate in the analysis of 1 or 2 unknown samples using their own methods. The aim was to compare the different analyses and improve the cooperation between the laboratories to optimise analytical techniques. Analysis of steviol glycosides is not easy and not only the HPLC methods used are critical as a baseline separation is required, but also the whole set of good laboratory practices (GLP). Different aspects of analysis have to be considered such as: drying of the samples and standards to a constant weight, the purity of the standards, the weighing process itself, the dissolution of samples and standards, the injection and daily calibration of the HPLC, the identification of different compounds and the integration of all peaks and correction for differences in molecular mass. The results presented in this paper are the data sent to us by the different participating laboratories. The results were treated anonymously, and each lab received a number to compare its own results with those of the other laboratories.

\section{Results}

\subsection{Theoretical Weight Loss and Purity of Sample 2 of the First Testing}

An interesting result from the first round-robin testing is the weight loss after drying to a constant weight. Heating $\mathrm{NaHCO}_{3}$ at $105^{\circ} \mathrm{C}$ causes a decomposition of $\mathrm{NaHCO}_{3}$ to $\mathrm{Na}_{2} \mathrm{CO}_{3}$ and loss of $\mathrm{CO}_{2}$ and $\mathrm{H}_{2} \mathrm{O}$ (total loss of $36.9 \%$ ). As sample 2 contained $80 \%$ of sample 1 and $20 \%$ of $\mathrm{NaHCO}_{3}$, the total loss by heating can be calculated as follows:

The weight loss of sample 1 (only steviol glycosides) is $6 \%$. If $100 \mathrm{mg}$ of ("wet") sample 2 is taken, it contains $80 \mathrm{mg}$ steviol glycosides and when heated at $105^{\circ} \mathrm{C}$, a loss of 4.8 $\mathrm{mg}$ can be expected (6\%) plus a $36.9 \%$ weight loss of $20 \mathrm{mg}$ bicarbonate $=7.38 \mathrm{mg}$. The total loss of $100 \mathrm{mg}$ of sample 2 is thus $4.8+7.38=12.18 \mathrm{mg}$ or $12.18 \%$. Dry matter content of sample 2 is thus $87.82 \%$. The reported loss on drying of sample 2 varied between $4.9 \%$ and $12.7 \%$. This demonstrates that not all the laboratories dried the sample to a constant weight.

The sample used in the second test had a purity of $91.1 \%$ and contained a mixture of 11 steviol glycosides. Below, only the results of the second round-robin testing are given.

\subsection{Construction of Calibration Curves}

Ultra-pure standards were prepared of the steviol glycosides [13] and calibration curves were made with ST, RebA and Rub. Drying to constant weight, weighing and handling were done using GLP. Automatic pipettes were avoided and solutions were made on a weight basis as this reduces possible errors (see below).

Figure 1 shows the calibration curves of ST, Reb A and Rub plotted as mM concentrations. Although the molecular 
weights were totally different $(804,966$ and 642 for ST, RebA and Rub, respectively), the slopes of the calibration curves plotted as $\mathrm{mM}$ concentrations were very similar (1429.6 \pm 26.9 ). However, use of isocratic solvents prolongs analysis time and, hence, the integration of small peaks eluting late might become problematic if calibration is done with early eluting compounds only. Therefore, solvent gradients were used.

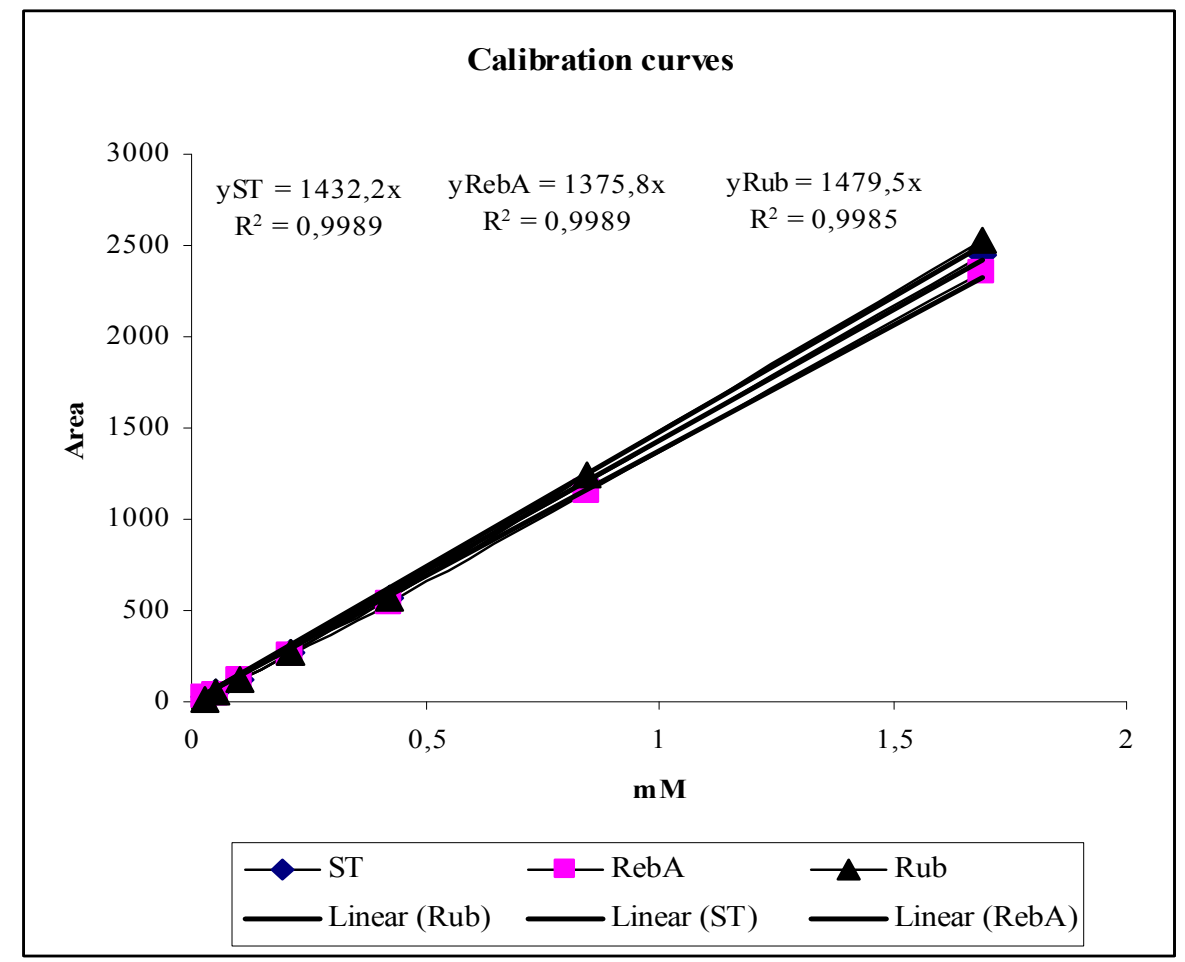

Figure 1. Calibration curves of ST, RebA and Rub plotted in mM concentrations.

$\mathrm{mV}$

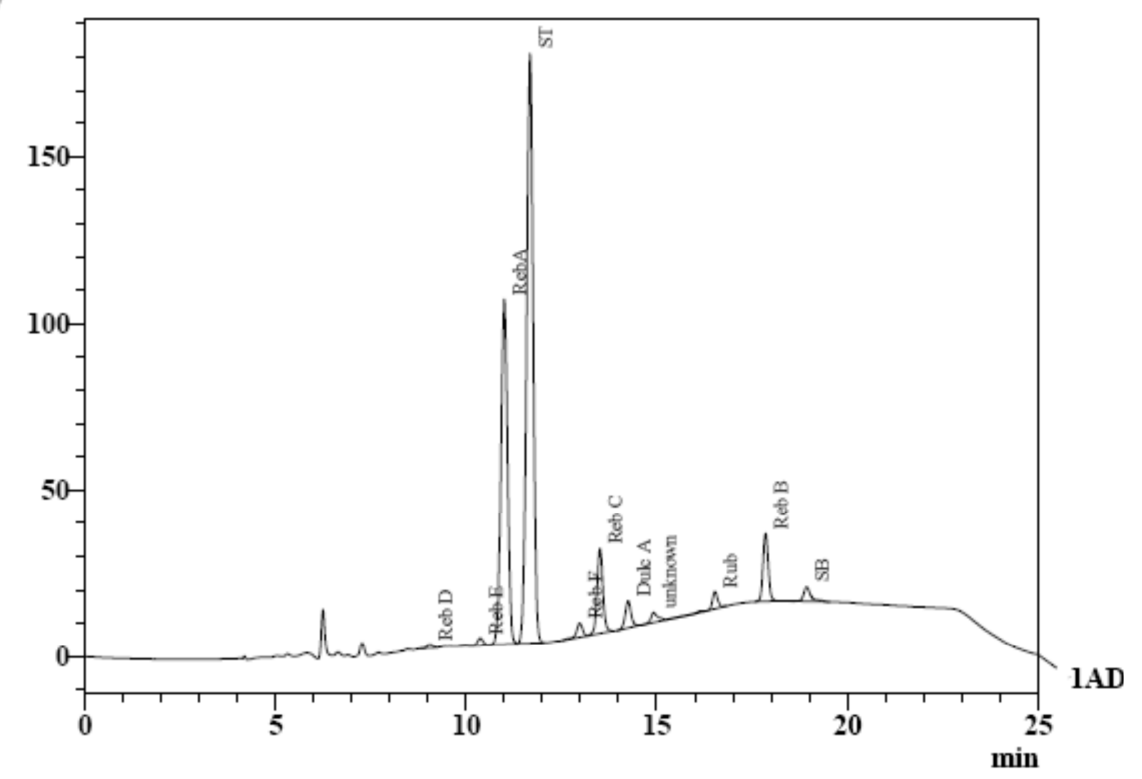

Figure 2. HPLC trace of the sample run on 2 Grace Alltima ODS-columns in series.

\subsection{Analysis of the Sample of the Second Testing}

A baseline separation was obtained by use of 2 Grace Alltima $\mathrm{C}_{18}$ columns in series and a solvent gradient of AcCN: $0.1 \mathrm{mM}$ phosphoric acid starting at 34: 66 (1 - $4 \mathrm{~min})$ going to 41.6 : 58.4 at $10 \mathrm{~min}$ (4.1 - $10 \mathrm{~min})$. After $6 \mathrm{~min}$ at this solvent strength $(10.0-16.0 \mathrm{~min})$, the columns are rinsed with the first solvent $(16.1-25 \mathrm{~min})$. Total analysis time is less than $30 \mathrm{~min}$ (Figure 2). The detection was at $197 \mathrm{~nm}$.

The average drying loss of the sample was about $4.4 \%$ $(n=6)$. Although the Karl Fischer method measures water content more precisely, this method is not retained as it is expensive. Moreover, JECFA [3] suggested that samples be 
dried to a constant weight.

Table 2 gives the analysis values by a validated external

standard method of the sample of the second round-robin testing.

Table 2. Percentage composition and $\mathrm{mg}$ of each component per $100 \mathrm{mg}$ dry wt. of the mixture (corrected for water loss, 4.4\%).

\begin{tabular}{llll}
\hline Name & Retention time(min) & \% of SVgly & mg/100 mg dry sample \\
\hline Reb D & 9.043 & 0.36 & 0.32 \\
Reb E & 10.37 & 0.58 & 0.53 \\
Reb A & 10.99 & 34.02 & 30.83 \\
ST & 11.73 & 47.75 & 43.26 \\
Reb F & 12.98 & 1.17 & 1.06 \\
Reb C & 13.51 & 7.16 & 6.48 \\
Dul A & 14.25 & 1.92 & 1.74 \\
Reb G & 14.92 & 0.87 & 0.79 \\
Rub & 16.53 & 0.86 & 0.78 \\
Reb B & 17.86 & 4.37 & 3.96 \\
SB & 18.94 & 0.94 & 0.85 \\
Purity & & & 90.6 \\
\hline
\end{tabular}

The results of the analyses of this sample by the different laboratories are given in Table 3. Most of the laboratories used $\mathrm{NH}_{2}$ columns, except those marked with an asterix $\left(*=\mathrm{C}_{18}: * *=\mathrm{HILIC}\right)$.

Table 3. Analyses of the sample by the participating laboratories. Values are expressed on a dry wt. basis.

\begin{tabular}{|c|c|c|c|c|c|c|c|c|c|c|c|c|c|}
\hline Lab & $\%$ loss & RebD & RebE & RebA & ST & RebF & RebC & DulA & RebG & Rub & RebB & SB & SM \\
\hline $2 * *$ & 3.64 & & & 30.82 & 45.22 & & 6.21 & 5.92 & & & & & \\
\hline $5^{*}$ & $?$ & & & 30.89 & 47.17 & 1.19 & 7.25 & 2.05 & 0.60 & 1.08 & 4.62 & 0.90 & 0.09 \\
\hline $6^{*}$ & 4.4 & 0.33 & 0.52 & 30.9 & 43.5 & 1.1 & 6.50 & 1.70 & 0.80 & 0.80 & 4.0 & 0.9 & \\
\hline 9 & 4.37 & 1.39 & & 31.22 & 43.8 & 1.05 & 6.27 & 1.31 & & 0.79 & 4.37 & 0.82 & \\
\hline 11 & 3.3 & & & 27.32 & 43.4 & & 5.51 & 1.24 & & 0.78 & 3.95 & 0.54 & \\
\hline 15 & 2.98 & & & 30.30 & 42.5 & 1.1 & 6.5 & 1.8 & 0.7 & 0.7 & 4.5 & 0.8 & \\
\hline 16 & 3.15 & & & 31.19 & 44.66 & 0.93 & 6.59 & 1.83 & 0.58 & 0.73 & 4.36 & 0.93 & \\
\hline
\end{tabular}

* Using $\mathrm{C}_{18}$ columns

** Using HILIC columns bold and italics: only percentage composition was given

Table 4 lists the purity of the sample as reported by the different laboratories. As some laboratories did not measure all the smaller peaks, values were added to obtain a "corrected purity".

Table 4. Purity of the sample obtained by different laboratories (only those reporting results).

\begin{tabular}{lllll}
\hline Lab & Purity (\% of dry wt) & \# compounds analysed & Correction from labs 5 and 6 & Corrected purity \\
\hline $2^{* *}$ & 88.2 & 4 & 8.54 & 96.74 \\
$5^{*}$ & 95.86 & 10 & 0.85 & 96.71 \\
$6^{*}$ & 91.1 & 117 & 0.09 & 91.19 \\
9 & 91.01 & 9 & 2.41 & 92.42 \\
11 & 82.74 & 7 & 0.94 & 85.58 \\
15 & 88.9 & 9 & 0.94 & 89.84 \\
16 & 91.8 & 9 & nd \\
17 & nd & 2 & & 92.74 \\
\hline
\end{tabular}

\section{Discussion}

One laboratory (17) analysed only the percentage composition of 2 SVgly and 1 laboratory (2) analysed only 4 compounds. The other laboratories analysed between 7 and 11 SVglycosides of the mixture. None of the laboratories reported the occurrence of SV, which is not expected in the mixture of these relatively polar SVgly. Only 1 lab (6) reported the values for Reb E $(0.52)$ and 2 laboratories $(6,9)$ the values for Reb D $(0.33,1.39)$. The values recorded on a dry wt. basis varied between 27.32 and 31.19 and between 42.5 and 47.17 for Reb $\mathrm{A}$ and ST, respectively. Reb F was reported by 5 laboratories and its value was between 0.93 and 1.19. Reb $\mathrm{C}$ varied between 5.51 and 7.25. A much larger spread was found for Dul A: between 1.24 and 5.92. An unidentified compound in the mixture with MW 804, tentatively named Reb G, was analysed by 4 laboratories (between 0.58 and 0.80 ). This compound is not yet fully characterised by NMR (Geuns, unpublished). The values for Rub, Reb B and SB were measured by 6 laboratories and their amounts varied between 0.7 and 1.08, 3.95 and 4.62 and between 0.54 and 0.93 for Rub, Reb B and SB, respectively. Only 1 laboratory (5) analysed SM (0.09). The weight loss after drying of the sample varied between $2.98 \%$ and $4.4 \%$ (not all laboratories reported the weight loss). The purity of the sample obtained by the addition of all compounds analysed, is given in Table 4 (second column). The purity of the sample was $91.1 \%$, checked by the 
standard addition method of 3 SVgly as described by [2] (Reb A, ST and Reb B). Three laboratories obtained a value of about the expected purity $(91.01-91.8)$. Laboratory 5 overestimated the purity $(95.86 \%)$ whereas the other laboratories reported purities between 88.2 and $88.9 \%$. As not all laboratories analysed all the compounds present in the mixture, a correction was made by adding the values of nonanalysed compounds taken from laboratories 5 (SM only) and 6. After the addition of the "lacking" values, the "corrected" purities varied between 85.58 and $96.74 \%$ (Table 2). In this way, 4 laboratories $(6,9,15$ and 16) obtained acceptable values only differing from the expected values by no more than $1.5 \%$. Laboratory (11) underestimated the purity and 2 labs overestimated the purity by about $6 \%$.

This second round-robin testing has shown that it is difficult to motivate the participating laboratories to send their results before the deadline although about 2 months were given for performing 1 analysis. However, there are a number of positive points to report. Most of the laboratories did analyse many more compounds than they did the first time. Moreover, the differences for the compounds analysed were less than during the first round-robin testing, certainly for the main compounds present. This can be evaluated by the values of the relative standard deviation (RSD, Table 5). The RSD's were calculated on the average values provided by the different laboratories. Reb D and E were not included as the number of measurements was too small. In the first roundrobin testing, the RSD's were always about 50\% larger than those calculated on the results of the second one. This means that the quality of the different analyses has improved.

Table 5. RSD calculated on the averages of the first and second round-robin testing of steviol glycosides.

\begin{tabular}{|c|c|c|c|c|c|c|c|c|c|c|}
\hline & RebA & ST & RebF & RebC & dulA & RebG & Rub & RebB & SB & $\%$ \\
\hline first & 12.7 & 6.4 & 24.5 & 19.5 & 38.1 & 8.3 & 2.4 & 87.5 & 84.2 & 5.6 \\
\hline second & 4.5 & 3.5 & 8.8 & 8.1 & 72.3 & 15.1 & 16.7 & 6.3 & 17.7 & 4.5 \\
\hline
\end{tabular}

The accuracy of analysis of the most important sweeteners (Reb A, ST, Reb C) was much improved too. The participants who participated for the first time had access to the guidelines of the previous testing [2]. This certainly helped them to improve their analysis. To further improve the quality of the analyses, the different laboratories should try to identify and quantify all the SVgly present in a sample. Probably 2 injections will be necessary to improve the quantification of the smaller peaks, one injection as usual, and one injection of a 5 or $10 \mathrm{x}$ more concentrated solution. To better dissolve the samples and to avoid precipitation, a good co-solvent might be required. This might pose problems with the external standard method that, so far, is used for the quantitation of SVgly, as small amounts of the co-solvent might evaporate, leading to faulty results. Impurities of the co-solvent might also show up.

The required purity of steviol glycosides is $95 \%$. The mean RSD of $4.5 \%$ obtained is still rather large, as it means that then purities of $95 \pm 4.5$ have to be accepted, i.e. purities between 90.5 and $99.8 \%$. Is it possible to increase the accuracy and to reduce the RSD? How can it be done?

The listing below is a non-exhaustive list of possible items enhancing the degree of errors influencing the accuracy of measurement of steviol glycosides by the external standard method.

Item

\section{External Standard Itself}

- Purity of standards:

High purity standards are required and were made [8]. The purity of commercial standards is sometimes far from the claimed purity values.

- Water content of standard:

Standards may contain up to $5 \%$ water and should be dried to a constant weight before use.

1) Weigh an empty and dry weighing vessel with lid (value A).

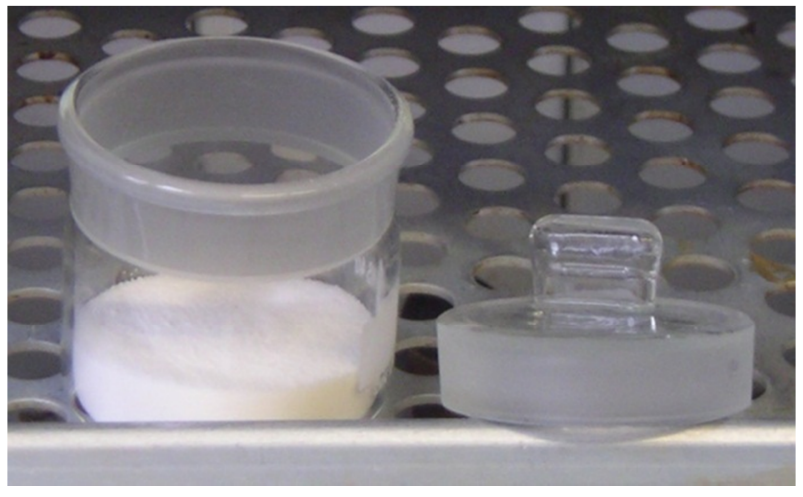

Figure 3. Example of a weighing vessel with lid also placed in the oven.

2) Exactly weigh about $500 \mathrm{mg}$ of the standard or unknown sample of SVglys in the weighing vessel with lid (value B).

3) The amount of wet sample is: $\mathrm{C}=\mathrm{B}-\mathrm{A}$.

4) Dry the opened vessel with wet mixture of standard or analyte to a constant weight or overnight $(16 \mathrm{~h}$ at $105^{\circ} \mathrm{C}$ ). Do not forget to place the lid in the oven to avoid expansion/contraction problems when cooling down the closed vessel.

5) After the drying period, place the lid on the hot vessel in the oven and allow it to cool in a desiccator for about $15 \mathrm{~min}$.

6) Weigh the vessel with dried sample (value D).

7) The dry weight of the unknown sample is $E=D-A$ (mg dry wt.).

8) The percentage dry weight is: $\mathrm{F}=\mathrm{E} / \mathrm{C} \times 100$ (times 100 to present it as a percentage).

9) The water content in percentage is: $\mathrm{G}=100-\mathrm{F}$.

- Weighing process of the standards:

A sufficient large amount of standard should be weighed to decrease the percentage of uncertainty. Even by using an analytical balance with a resolution of $\pm 0.1 \mathrm{mg}$, weighing only 
$2 \mathrm{mg}$ gives an uncertainty of $10 \%$, i.e., $2 \mathrm{mg} \pm 0.1 \mathrm{mg}$ or $(0.2 / 2)$ x $100=10 \%$. To reduce the possible error beneath $1 \%$, at least $50 \mathrm{mg}$ of standard should be weighed $(0.2 / 50) \times 100=0.4 \%$. Taking each time $50 \mathrm{mg}$ of very pure standards of the compounds occurring in small amounts would become very expensive, and reuse of standards might introduce impurities. For this reason, preliminary research has been done and it was shown that calibration with only 1 standard is sufficient for the measurement of steviol glycosides (see Figure 1). Calibration curves should be made in $\mathrm{mM}$ concentrations.

- Calibration solution of standard:

Calibration curves should be made on a weight basis starting with at least $50 \mathrm{mg}$ of the standard. Much uncertainty can be introduced even by using calibrated pipettes as observed by the author who unexpectedly tested calibrated pipettes in an analytical laboratory by weighing $1 \mathrm{ml}$ water and found deviations up to $10 \%$ (results not presented). If pipetting of standards would be required, this should be done in syringes with the volume to be delivered trapped between 2 air bubbles (cfr. methods for quantitative injection in GC). This way the exact volume can be checked and the syringe is rinsed with solvent that was first sucked into the syringe.

Analyte

- Drying process: the product should be dried to a constant weight (see above)

- Weighing: at least $50 \mathrm{mg}$ should be weighed to avoid weighing errors (see above). In case of analysis of plant material (leaves) at least $200 \mathrm{mg}$ should be taken for analysis to avoid a bad sampling. In many papers in the scientific literature, only 20 mg leaf tissue is analysed and this is a too small amount as 20 mg might come from only 1 leaf. It is known that leaf position on the plants has a huge influence on the steviol glycoside amount [14]. The analysis of only $20 \mathrm{mg}$ leaf tissue might also over- or underestimate the yield per hectare when results of this kind of analyses are extrapolated to know the yield per ha.

- Purification of crude extracts:

Purification of water extracts by SPE techniques is certainly advantageous for maintaining good column conditions. However, each purification step can add a degree of uncertainty to the analysis as losses will certainly occur. Several SPE methods are given for the purification of different extracts of food samples [11].

Analysis

- The injection volume of standard or analyte is very critical, even with an automatic injector. This can be checked by injection of different volumes of water and collecting and weighing the volume that was delivered by the injector. In this way, possible errors can also be detected if different amounts would be injected to increase the size of small peaks (e.g., 20 $\mu \mathrm{l}$ versus $100 \mu \mathrm{l}$ injections).

- Change of sensitivity of the detector:

The sensitivity of a detector might change after the light bulb has been used for a while. This should be checked at regular moments.

- Dissolution of analyte:

Some compounds of the mixture might be less soluble and be extruded from the mixture. Adding a solvent (e.g. EtOH or
$\mathrm{MeOH}$ ) might prevent this, but part of the $\mathrm{EtOH}$ or $\mathrm{MeOH}$ might evaporate from the injection vials and this might contribute to errors.

- Intra-laboratory and Inter-laboratory RSD of the 10 components of the mixture should be as small as possible and for the required purity level of steviol glycosides $(\geq 95 \%)$ should be below $2 \%$. This way, a claimed purity of $95 \%$ would lie between 93 and 97\% which seems acceptable.

Volume Aspects that might Influence Accuracy

- If there is no control on the temperature of the samples to analyse, expansion or contraction of solvent (e.g., water) might increase uncertainty. This can give changes of sample and injection amounts.

- Inaccuracy of pipettes: pipettes used are possibly not recently calibrated and solvent expansion or contraction also has an influence. Therefore, weighing of all solvents is the best way of handling this problem.

- Precipitation of analyte: when concentrated solutions are used, some compounds might precipitate. The addition of cosolvents might help reduce this problem (keep out for evaporation, see above).

- Daily calibration necessary:

The external standard method requires a daily calibration of the whole equipment and this is time-consuming and expensive (very pure standards are required).

- Calculation errors possible:

Even with well-trained personnel, calculation errors are possible and all analyses should be double-checked to avoid human errors.

- Small peaks:

Small peaks make peak integration very difficult and erroneous. The injection of $5 \mathrm{x}$ more sample is not always possible, the more as then new calibration curves have to be made for the higher amounts injected. It cannot be done to inject $5 \mathrm{x}$ more and divide then the peak areas by 5 . This would introduce more uncertainty. The best way of handling is to make solutions that are $5 \mathrm{x}$ more concentrated and stick to the same injection volume. However, then there might be a problem of precipitation of some less soluble material. Adding a co-solvent might circumvent this, but then problems of evaporation might occur or additional peaks appearing in the chromatogram due to impurities in the co-solvent.

Stress Factor of the Personnel

Although the personnel of the laboratory were well trained, the huge list of demands for perfect performances throughout steviol glycoside analysis nearly drove them to madness. Therefore, an internal standard method should be developed that will prove its value, not only for solvent losses as above, but also for losses of sample during sample clean-up when extracting complex food matrices, as well as for differences in injection volume and a possible change of detector sensitivity. Work is in progress to circumvent all the problems of an external standard method. However, for the moment being, when people are following the above guidelines, nearly perfect analyses can be obtained as shown by 4 laboratories that reported results only differing by $1.5 \%$ from the target value. 


\section{Conclusion}

JECFA [3] suggested the use of reverse phase columns for the analysis of steviol glycosides. A baseline separation of the most important steviol glycosides can be obtained by use of 2 reverse phase columns in series (e.g., 2 Grace Alltima $\mathrm{C}_{18}$ columns) and a solvent gradient of $\mathrm{AcCN}$ : $0.1 \mathrm{mM}$ phosphoric acid starting at 34: $66(1-4 \mathrm{~min})$ going to $41.6: 58.4$ at $10 \mathrm{~min}$ (4.1 - $10 \mathrm{~min})$. After $6 \mathrm{~min}$ at this solvent strength (10.0 - 16.0 $\mathrm{min})$, the columns are rinsed with the first solvent $(16.1-25$ min). Total analysis time is less than $30 \mathrm{~min}$ (Figure 2). The detection can be done at $200 \mathrm{~nm}$ if the equipment is suited for this. Recently, the use of 1 ultra-high performance liquid chromatography column was reported also giving a baseline separation [6]. Without baseline separation, the peak integration becomes more difficult and it might become impossible to obtain an inter-laboratory RSD of around $1 \%$. The correct analysis of steviol glycosides is a real challenge for the analytical chemist and many pitfalls exist. Taking into account all the above suggestions and GLP, it might be possible to do correct steviol glycoside analyses by an external standard method. Then an inter-laboratory RSD of about $1.5 \%$ can be obtained as shown by 4 laboratories that took care of all the suggestions made. In this way, a purity of $95 \%$ [15] means that the interval of uncertanty is rather small $(95 \%$ purity means than that values between 93.5 and $96.5 \%$ might be accepted). However, the analysis remains a difficult issue and the use of an internal standard might eliminate most of the tedious points in the analysis.

\section{Acknowledgements}

The author acknowledges the skilful technical assistance of Hilde Verlinden and Tom Struyf as well as the financial support by Medherbs, Wiesbaden, Germany, and Stepaja, Aarschot, Belgium. None of the funding organisations had any role in the design and conduct of the study; collection, management, analysis, and interpretation of the data; and preparation, review, or approval of the manuscript.

\section{Abbreviations}

Using SV for steviol allows the use of the following abbreviations: SVgly: steviol glycosides; SVeq: steviol equivalents; SVglu: steviol glucuronide; SM: steviol monoside; SVE: steviol 19-ester; SVglu: steviol glucuronide; ST: stevioside, RebA - G: rebaudioside A - G; SB: steviolbioside; DulA: dulcoside A; Rub: rubusoside.

\section{References}

[1] Geuns J.M.C. and Struyf T.: EUSTAS Round-Robin Testing of Steviol Glycosides pp. 35-48 in Proceedings of the $3^{\text {rd }}$ EUSTAS Stevia Symposium "Stevia in Europe" July $1^{\text {st }}-2^{\text {nd }}$ 2009, KULeuven, Belgium Ed.: Jan M.C. Geuns, ISBN: 97890-742-53079.

[2] Geuns J.M.C.: Stevia and steviol glycosides. (2010) Euprint
Ed., Parkbosstraat 3, 3001 Heverlee, Belgium, ISBN: 9789074253116, pp. 307.

[3] JECFA (2010) Steviol glycosides. FAO JECFA Monograph 10 .

[4] Scaglianti M., C. Gardana, P.G. Pietta and G.M. Ricchiuto (2008) Analysis of the main Stevia rebaudiana sweeteners and their aglycone Steviol by a validated LC-DAD-ESI-MS method. pp. 45-58 in Proceedings of the EUSTAS Stevia Symposium, June $27^{\text {th }} 2008$, KULeuven, Belgium Ed.: Jan M.C. Geuns, ISBN: D/2008/6045/50.

[5] Zimmermann B.F. and U. Wölwer-Rieck (2011) HILIC columns for the analysis of steviol glycosides. pp. 147-152 in Proceedings of the $5^{\text {th }}$ EUSTAS Stevia Symposium Stevia, Breakthrough in Europe, June 28-29, 2011, KULeuven, Belgium Ed.: Jan M.C. Geuns, ISBN: 978-90-742-53192; $\mathrm{D} / 2011 / 6045 / 038$.

[6] Zimmermann B.F. (2018) Beaming steviol glycoside analysis into the next dimension. Food Chemistry, 241, 150-153.

[7] Chatervedula, V. and Zamora J. (2014) Reversed-phase HPLC analysis of steviol glycosides isolated from Stevia rebaudiana Bertoni. Food and Nutrition Sciences 5, 1711-1716.

[8] Tada A., Ishizuki K., Mikami H., Hirao Y., Fujita L. et al. (2013) Improvement of the assay methodo for steviol glycosides in the JECFA specifications. American Journal of Analytical Chemistry 4, 190-196.

[9] Soufi S., D’Urso G., Pizza C., Rezgui S, Bettaieb T and Montoro P (2016) Steviol glycosides targeted analysis in leaves of Stevia rebaudiana (Bertoni) from plants cultivated under chilling stress conditions. Food Chemistry 190(C), 572580 .

[10] Molina-Calle M., Sánchez de Medina V., Delgado de la Torre M.P., Priego-Capote F. and Luque de Castro M.D. (2016) Development and application of a quantitative method based on LC-QqQ MS/MS for determination of steviol glycosides in Stevia leaves. Talanta 154(C), 263-269.

[11] Geuns J.M.C. (2008) Analysis of Steviol glycosides: validation of the methods. pp. 59-78 in Proceedings of the EUSTAS Stevia Symposium, June $27^{\text {th }} 2008$, KULeuven, Belgium Ed.: Jan M.C. Geuns, ISBN: D/2008/6045/50.

[12] Struyf T., Chandia N.P., De Borggraeve W., Dehaen W. and Geuns.J.M.C.: Preparation of pure standards of steviol glycosides. Identification of steviol glycosides by LC-MS and NMR. pp. 29-44 in Proceedings of the EUSTAS Stevia Symposium, June $27^{\text {th }} 2008$, KULeuven, Belgium Ed.: Jan M.C. Geuns, ISBN: 9789074253-031; D/2008/6045/50.

[13] Kumari N. and Kumar S. (2017) Chemistry and analytical techniques for ent-kaurene-glycosides of Stevia rebaudiana Bertoni - A review. Journal of Applied and Natural Sciences 9 (4), 2114-2126.

[14] Ceunen S. and Geuns J.M.C. (2013) Influence of photoperiodism on the spatio-temporal accumulation of steviol glycosides in Stevia rebaudiana (Bertoni). Plant Science 198, 72-82.

[15] EFSA (2011) EU Commission. Commission Regulation (EU) No. 1131/2011. Official J. Eur. Union 2011, L295, 205-211. 